\title{
BRIOZOOS FENESTRADOS DE LA FORMACIÓN MONIELLO (DEVÓNICO) EN EL ÁREA DE ARNAO (ASTURIAS, ESPAÑA). I: FENESTELLIDAE
}

\author{
Juan Luis SUÁREZ ANDRÉS
}

Universidad de Oviedo, Departamento de Geología, Área de Paleontología. Arias de Velasco s/n. 33005 Oviedo (Asturias).

\begin{abstract}
Suárez Andrés, J.L. 1998. Briozoos Fenestrados de la Formación Moniello (Devónico) en el área de Arnao (Asturias, España). I: Fenestellidae. [Fenestrate Bryozoa from the Moniello Formation (Devonian) in Arnao (Asturias, Spain). I: Fenestellidae]. Revista Española de Paleontología, 13 (2), 187-196. ISSN 0213-6937.
\end{abstract}

\begin{abstract}
A first approach is made to the systematics of the Devonian Fenestrates from the Cantabrian Zone (NW Spain), focused on the upper member of the Moniello Formation (late Emsian-early Eifelian). In this work, the representatives of the fenestrate family Fenestellidae are described; the remaining taxa will be treated in a following paper. This family seems to be the most diverse in the outcrop, with four species here described: Cyclopelta winteri, Cyclopelta sp. A, Semicoscinium cf. discretum and Hemitrypa sp. A. Besides, other species assigned to these genera are being studied, and some Fenestella-like forms occur, that still are to be analyzed. The systematic study has been carried out following the methodology developed by Snyder (1991a, b).
\end{abstract}

Keywords: Bryozoans, Fenestellidae, Devonian, Asturias, Spain.

\section{RESUMEN}

Se realiza por primera vez para el Devónico de la Zona Cantábrica un estudio sistemático de los briozoos Fenestrados, centrado en el miembro superior de la Formación Moniello (Emsiense superior-Eifeliense inferior). En el presente trabajo se describen los representantes de la familia Fenestellidae; los demás taxones serán tratados en una próxima publicación. Esta familia parece ser la más ampliamente representada, con cuatro especies pertenecientes a tres géneros: Cyclopelta winteri, Cyclopelta sp. A, Semicoscinium cf. discretum y Hemitrypa sp. A. Existen además otras especies asignadas a estos géneros que están siendo estudiadas, así como varias formas relacionadas con el género Fenestella que aún no han sido analizadas. El estudio sistemático se ha realizado siguiendo la metodología propuesta por Snyder (1991a, b).

\section{Palabras clave: Briozoos, Fenestellidae, Devónico, Asturias, España.}

\section{INTRODUCCIÓN}

Este trabajo representa una primera aproximación a la sistemática de los briozoos del Devónico en la Zona Cantábrica, los cuales nunca habían sido estudiados en detalle. De hecho, las citas son escasas y se encuentran en trabajos geológicos de carácter más o menos general, como los de Barrois (1882) y Comte (1959). Méndez Bedia (1976) y Arbizu el al. (1979) mencionan la presencia de briozoos en la Formación Moniello, y Álvarez Nava y Arbizu (1986) utilizan para sus estudios paleoecológicos sobre la Formación Aguión varios géneros de Fenestrados. Para el presente estudio se ha levantado una columna estratigráfica parcial (Fig. 1) y se ha realizado un muestreo exhaustivo de los briozoos fundamentalmente en el miembro superior de la
Formación Moniello (Emsiense superior-Eifeliense inferior) en el área de Arnao (Asturias). En dicho miembro predominan los representantes del Orden Fenestrida y del Suborden Fistuliporina (Fistulipora); son menos abundantes los Criptostomados (Acanthoclema, Intrapora), los Trepostomados (Stereotoechus) y los Tubuliporados (Hederellidae). Además de estos géneros, se han encontrado otras formas cuyo estudio aún no ha podido ser abordado. En cuanto a los Fenestrados, se han identificado nueve especies pertenecientes a siete géneros, la mayoría de los cuales se citan por primera vez en la Zona Cantábrica, y quedan aún por determinar especies de estos y otros géneros. El presente trabajo está dedicado a los representantes de la familia Fenestellidae identificados hasta el momento, que son los siguientes: Cyclopelta winteri, Cyclopelta sp. A, Semicoscinium cf. 


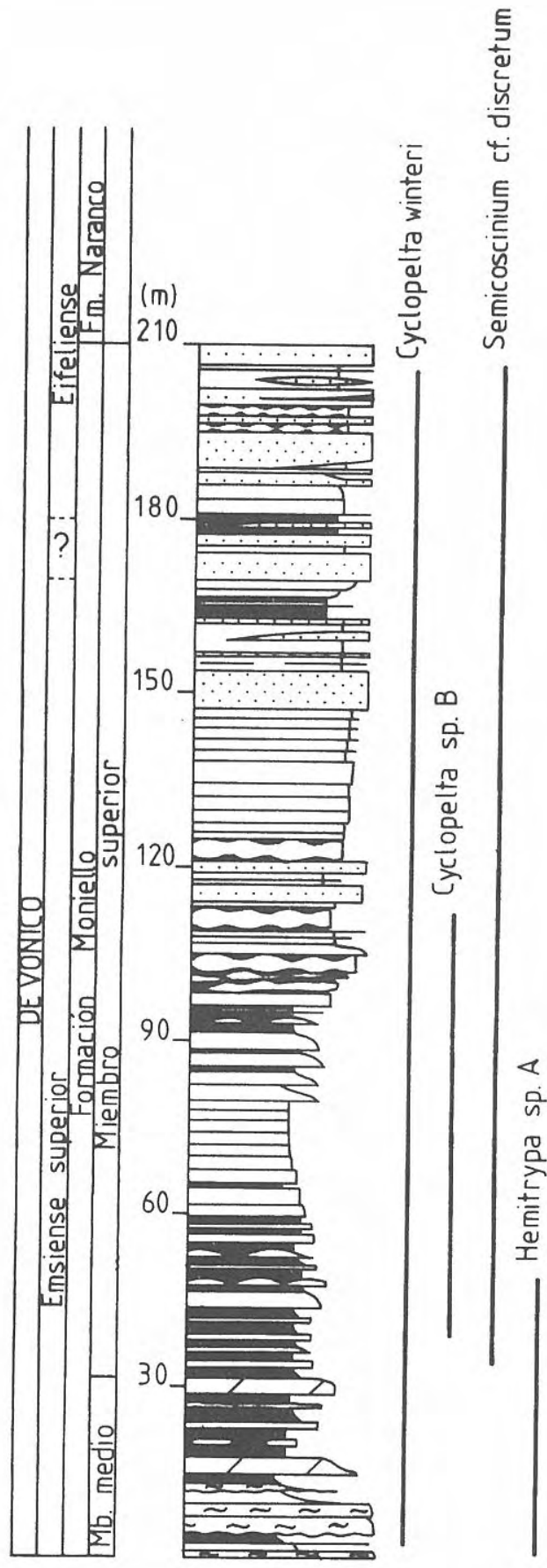

Marga

Caliza biostromal

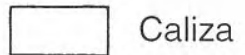

$\because \because \quad$ Caliza encrinítica

$\sim$ Tempestita

$\because$ Arenisca

Figura 1. Columna estratigráfica parcial de la Formación Moniello entre El Pical y la Playa de Arnao (Fig. 2 C), con la distribución de las especies de Fenestéllidos identificadas. discretum y Hemitrypa sp. A. Las especies analizadas que pertenecen a otras familias serán tratadas en un próximo trabajo, así como las consideraciones paleobiogeográficas que se pueden extraer de la fauna estudiada.

\section{CONSERVACIÓN Y DEPÓSITO DEL MATERIAL}

El material recolectado comprende unos 100 zoarios o fragmentos de zoarios, aunque su número exacto es difícil de precisar debido a la gran cantidad de ellos que se encuentran inmersos en la matriz rocosa. De todos modos, la especie más frecuente es Semicoscinium cf. discretum, seguida de Cyclopelta winteri y C. sp. A, también comunes a lo largo del corte. Hemitrypa sp. A no es abundante y sólo aparece con relativa frecuencia en los tramos con mayor abundancia y diversidad de briozoos. Si bien la mayor parte de las muestras se conservan en carbonato cálcico, en algunos casos las estructuras internas aparecen difuminadas por la recristalización, y es frecuente una silicificación parcial que a menudo afecta preferentemente al reverso de los especímenes. Los ejemplares están depositados en el Área de Paleontología, Departamento de Geología de la Univesidad de Oviedo. Cada especimen se identifica por unas siglas, PIC, referentes al yacimiento, y dos números; el primero indica el nivel de muestreo y el segundo el número de muestra correspondiente a dicho nivel.

\section{SITUACIÓN DEL ÁREA ESTUDIADA}

El yacimiento estudiado está comprendido entre la Punta del Pical y la Playa de Arnao, en la localidad de Arnao (Asturias), coordenadas $x=5^{\circ} 58^{\prime} 30^{\prime \prime}, y=43^{\circ} 34^{\prime} 50^{\prime \prime}$, de la Hoja 13 (Avilés) del mapa geológico de España, escala 1: 50.000 del IGME. Este afloramiento se encuentra en la Zona Cantábrica (Fig. 2), en la parte más septentrional de la Unidad de Somiedo-Correcilla, la más interna estructuralmente de la Región de Pliegues y Mantos (Julivert, 1967). Se trata del afloramiento más occidental de la Formación Moniello, que aparece afectada por cabalgamientos que enmascaran su espesor real. En el área de Arnao, los materiales pre-hercínicos se encuentran por lo general cubiertos discordantemente por depósitos estefanienses y mesozóicos (García Alcalde, 1992), aflorando únicamente en la costa.

\section{ESTRATIGRAFÍA}

La Formación Moniello comprende una sucesión de calizas y margas con ocasionales niveles pizarrosos, con un espesor que oscila entre 200 y $300 \mathrm{~m}$ y cuya edad es Emsiense superior-Eifeliense inferior. En esta unidad se distinguen tres miembros: el inferior, con unos $90 \mathrm{~m}$ de calizas y lutitas con braquiópodos; el medio, constituido generalmente por $60 \mathrm{~m}$ de calizas arrecifales, y el miembro superior, que suele estar formado por calizas y margas con fauna de braquiópodos y briozoos. El espesor de este último 


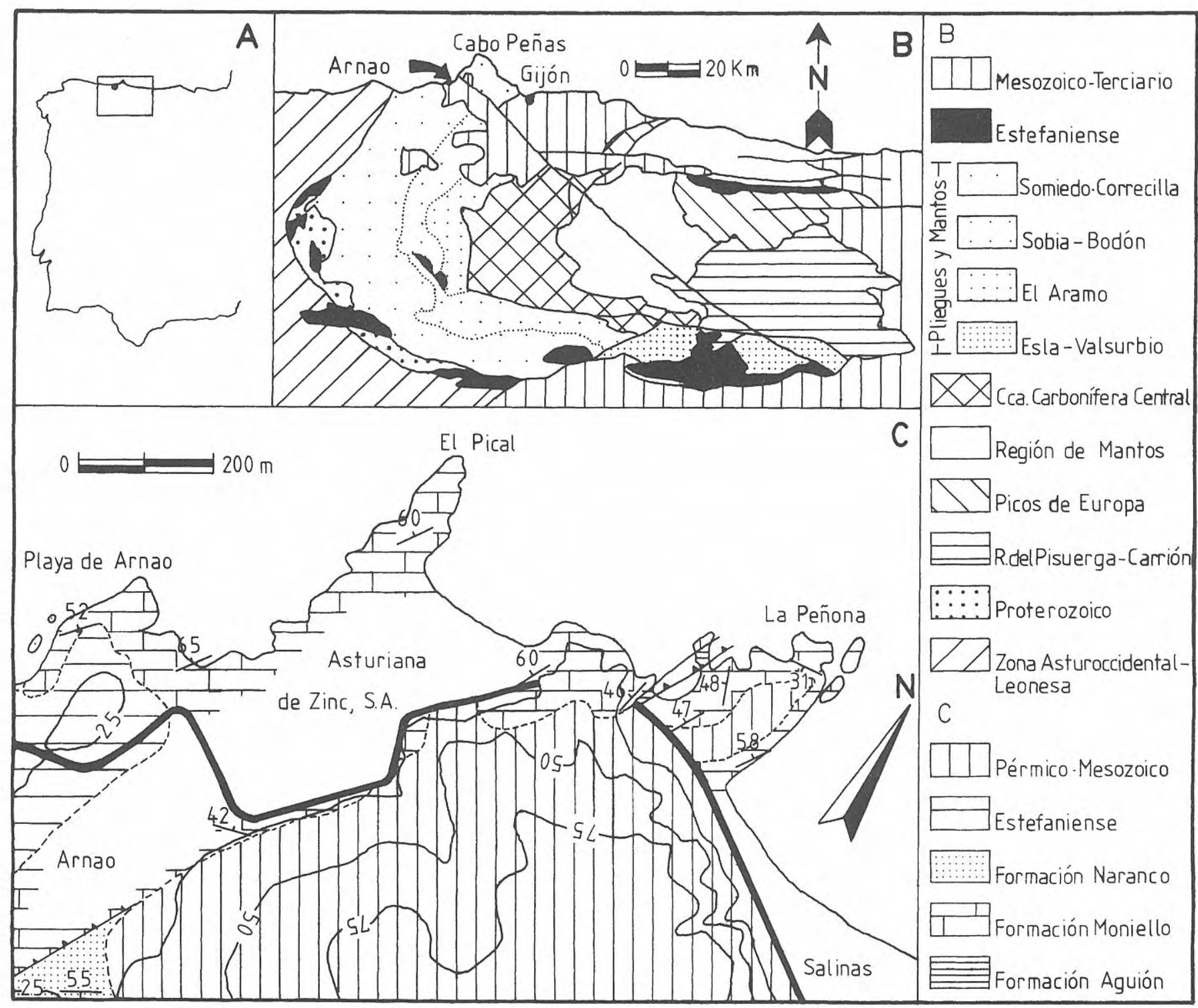

Figura 2. Situación geográfica y geológica del área estudiada. A: localización del yacimiento en la Península Ibérica. B: esquema simplificado de la Zona Cantábrica, indicando la situación de Arnao. C: mapa geológico de los alrededores de Arnao. El tramo estudiado, al que corresponde la columna estratigráfica (Fig. 1) va desde El Pical a la Playa de Arnao.

miembro suele ser de $120 \mathrm{~m}$, si bien en Arnao presenta un espesor anormal, como se observa en la columna estratigráfica (Fig. 1). Lo mismo ocurre con el miembro medio, compuesto por niveles biostromales y un arrecife biohermal de $140 \mathrm{~m}$ (Soto et al., 1994). Los estudios bioestratigráficos (Arbizu et al., 1979; Truyols et al., 1990; García Alcalde, 1995) sitúan el límite Emsiense-Eifeliense dentro del miembro superior, basándose en la fauna de braquiópodos, trilobites, corales, tentaculites y especialmente conodontos. Durante la realización de la cartografía (Fig. 2C) no se han encontrado estructuras tectónicas que expliquen el espesor del miembro superior de la Formación Moniello en la localidad estudiada. El hecho de que se trate de las facies más distales dentro de la plataforma hace pensar en una posible causa sedimentológica para este espesor, ya que las condiciones del medio permitirían acumular y conservar más cantidad de sedimentos en este lugar. No se ha investigado a este respecto por salirse del objetivo prioritario del trabajo.

\section{TÉCNICAS DE ESTUDIO}

Para una correcta determinación de los Fenestrados es necesario realizar tres secciones, orientadas tangencial, longitudinal y transversalmente al zoario, que permiten observar las características zoeciales y zoariales internas. En muestras silicificadas y recristalizadas la destrucción de la estructura interna obliga a realizar las determinaciones basándose en los caracteres externos, lo cual puede llevar a confusión, dada la naturaleza variable de algunos de ellos incluso para el mismo zoario. Se han realizado réplicas de acetato de las secciones orientadas, siempre que la conservación del material lo ha permitido. Se ha seguido 
básicamente el procedimiento propuesto por Boardman y Utgaard (1964), excepto en la utilización de un papel de acetato fino que debe ser montado entre dos vidrios para la conservación y manejo de las réplicas. Las medidas de los caracteres morfológicos se han realizado sobre fotografías de superficies zoariales y de las réplicas de las secciones. Snyder (1991a, b) desarrolló una metodología de estudio basada en la utilización de más de cuarenta caracteres, que permite una caracterización de los taxones muy precisa. Para cada carácter, el autor realiza 24 medidas y calcula la media, desviación estándar, rango y coeficiente de variación de dichos valores. Finalmente, acompaña las descripciones con los resultados tabulados del tratamiento estadístico, esquemas de secciones longitudinal, transversal y tangencial representativas de la especie y reconstrucciones tridimensionales de la cámara zoecial. Hageman (1991) valora las ventajas del método propuesto por Snyder (op. cit.), y demuestra que es posible obtener resultados casi igualmente satisfactorios utilizando diez o menos caracteres escogidos. Para el presente estudio se ha prescindido de los esquemas de las secciones y las reconstrucciones de los zoecios, y se ha utilizado una lista reducida de caracteres, cuyo número varía ligeramente en función de cada taxón. El valor de los resultados estadísticos es orientativo, ya que el número de ejemplares utilizados ha sido siempre inferior a diez. Se ha fijado en doce el número de medidas realizadas para cada carácter, indicándose en las tablas aquellos casos en los que no se ha podido completar este número.

\section{CARACTERES UTILIZADOS}

Se explica a continuación el significado de las abreviaturas utilizadas para los caracteres morfométricos en las tablas. Para la definición de dichos caracteres nos remitimos a los trabajos de Hageman (1991) y Snyder (1991a, b). ER: espesor de la rama; AR: anchura de la rama; LF: longitud de la fenéstrula; AD: anchura de disepimento; DCR: distancia entre centros de ramas; DAH: distancia entre centros de aberturas en una hilera; DAR: distancia entre centros de abertura a través de una rama; AA: anchura de la abertura zoecial; MAC: máxima anchura de la cámara zoecial; PC: profundidad de la cámara zoecial; LC: longitud de la cámara zoecial; ARC: ángulo agudo entre la lámina basal y la pared zoecial transversal; ARS y LRS: anchura y longitud de retícula, respectivamente (exclusivos de la superestructura reticulada de Hemitrypa). PC y LC se utilizan sensu Hageman (op. cit.).

\section{SISTEMÁTICA}

\author{
FILO BRYOZOA Ehrenberg, 1831 \\ CLASE STENOLAEMATA, Borg, 1926 \\ ORDEN FENESTRIDA Elias y Condra, 1957 \\ Familia Fenestellidae King, 1849 \\ Cyclopelta Bornemann, 1884
}

Especie tipo: Cyclopelta winteri Bornemann, 1884.

\section{Diagnosis}

Zoario en forma de embudo con el anverso situado en la superficie externa. Disepimentos alineados transversalmente a las ramas y espesados hacia el reverso, hasta formar anillos en el interior del embudo. Dos hileras de zoecios en cada rama, separadas por una quilla alta, bifurcada apicalmente. Pared axial sinuosa o en zigzag; parte alta de las quillas libre; sólo en ocasiones aparecen unidas por puentes. Zoecios pentagonales en sección tangencial profunda, más raramente rectangulares o hexagonales. Ramas y zoecios ensanchados en los puntos de inserción de los disepimentos. (Basada en Kräusel, 1953).

\section{Cyclopelta winteri Bornemann, 1884 Lám. I, figs. 1-3}

\section{Descripción}

Zoario en forma de embudo muy estrecho; algunos ejemplares conservan una región distal bruscamente ensanchada. La región proximal puede presentar pliegues longitudinales que llegan a estrangularse dando lugar a embudos laterales. Estas partes proximales suelen aparecer totalmente rellenas por tejido vesicular desde el interior del embudo al extremo de las quillas (Lám. I, figs. 2, 3). Anverso situado en el exterior del embudo. Ramas rectas, separadas entre sí por disepimentos anchos y cortos, alineados. Disepimentos espesados hacia el reverso hasta sobrepasar la altura de las ramas, uniéndose lateralmente para formar anillos concéntricos en el interior del embudo, característicos del género (subestructura de Kräusel, 1953, 1954). Fenéstrulas de tamaño medio, elípticas, selladas por tejido vesicular en las partes proximales de los zoarios. Disepimentos inclinados hacia el interior del embudo en sentido distal, proyectándose sobre las fenéstrulas

\section{Lámina I}

1-3 Cyclopelta winteri Bornemann, 1884. PIC-61-2; 1: sección tangencial mostrando cámaras y aberturas; en la parte inferior derecha se observa uno de los puentes que conectan las quillas. x24. 2: sección transversal; las dos quillas centrales están unidas por un puente, x26. 3: sección longitudinal; se observa la morfología de los disepimentos, las cámaras y la quilla; el tejido vesicular rellena desde el interior hasta el techo de la quilla, x26.

4-6 Cyclopelta sp. A. PIC-46.2-1; 4: sección tangencial mostrando la morfología de las cámaras, x22. 5: sección transversal, x28. 6: sección longitudinal, cortando

cámaras zoeciales con sus aberturas. El tejido vesicular recubre el anverso obstruyendo parcialmente las fenéstrulas, $\mathrm{x} 26$.

7 Cyclopelta sp. A. PIC-46.2-2; sección longitudinal mostrando la morfología y orientación de disepimentos y fenéstrulas respecto de las ramas, x26.

8 Semicoscinium cf. discretum (Prantl, 1932). PIC-69-4, sección tangencial cortando ramas a distintos niveles; destaca la pared axial, recta excepto en la parte superior derecha, y la morfología de las cámaras, x14. 
Lámina I

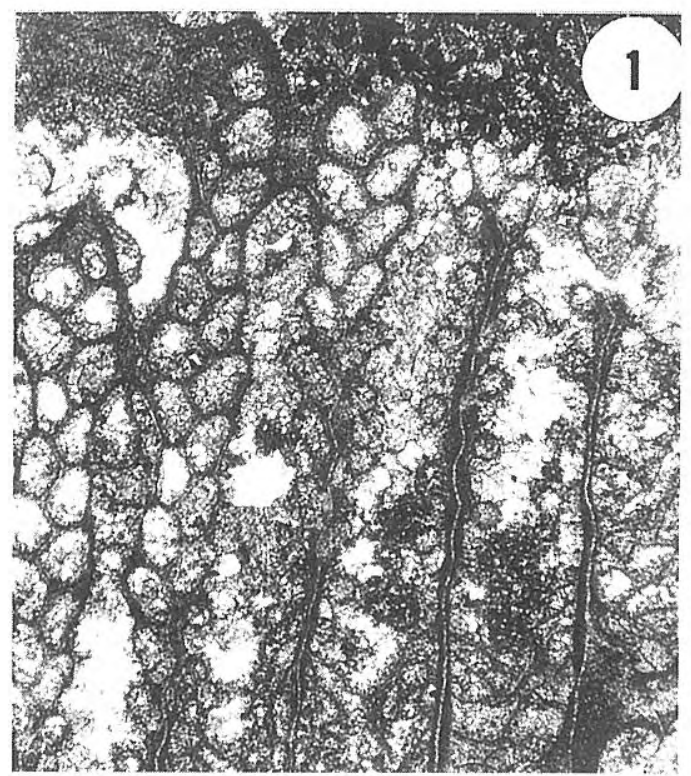

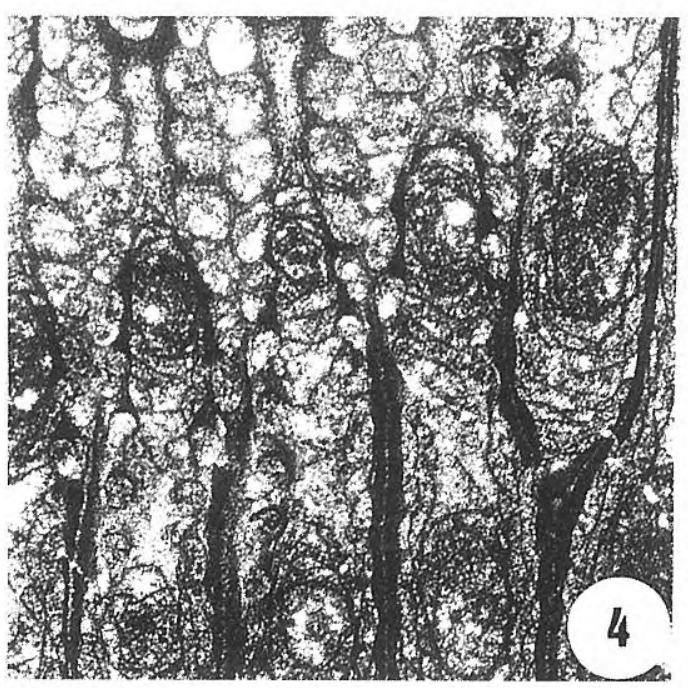

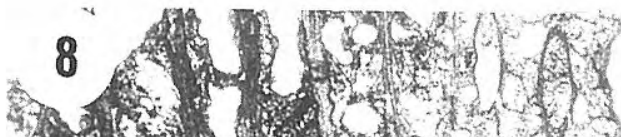

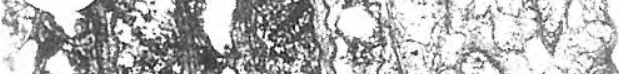

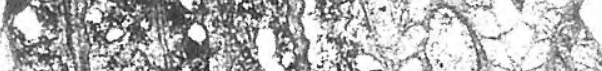

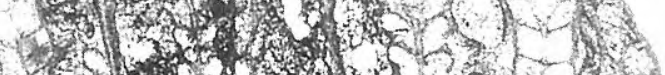
2.7.

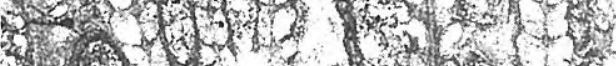

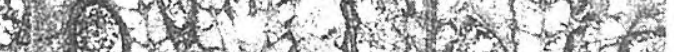

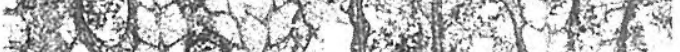

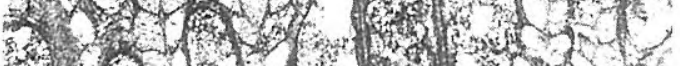
H.

$3(x)$ a Whit 1. 1 . (1)

Revista Española de Paleontología, 13 (2), 1998.
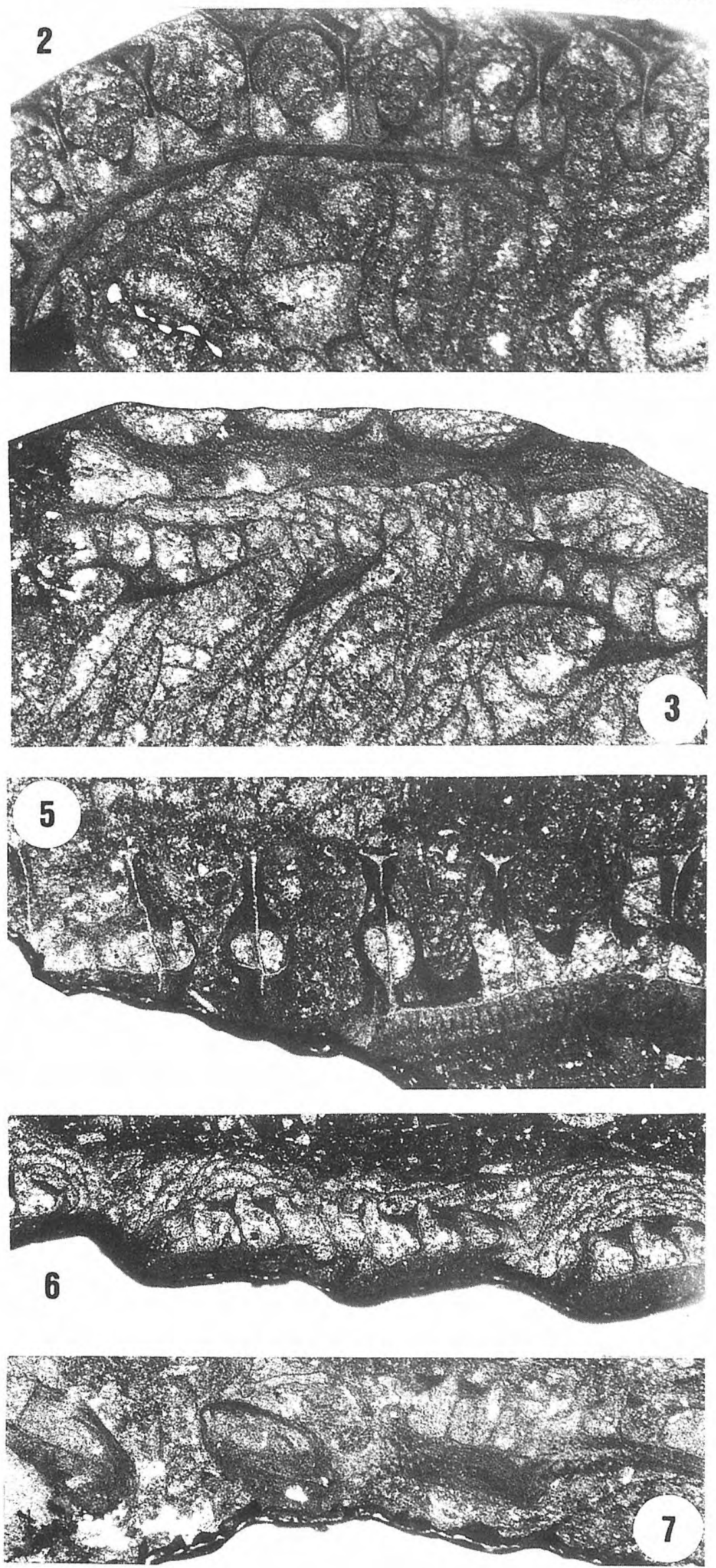


\begin{tabular}{|l|l|l|l|l|}
\cline { 2 - 5 } \multicolumn{1}{c|}{} & media & desviación & rango & cf. varianza \\
\hline ER & 0,9942 & 0,0328 & $0,94-1,05$ & 3,30 \\
\hline AR & 0,4058 & 0,0465 & $0,30-0,47$ & 11,46 \\
\hline LF & 0,7266 & 0,0403 & $0,67-0,76$ & 5,55 \\
\hline AD & 0,4375 & 0,0650 & $0,35-0,50$ & 14,86 \\
\hline DCR & 0,6125 & 0,0398 & $0,50-0,66$ & 6,50 \\
\hline DAH & 0,2696 & 0,0243 & $0,235-0,32$ & 9,01 \\
\hline DAR & 0,2796 & 0,0233 & $0,22-0,30$ & 8,33 \\
\hline AA & 0,1129 & 0,0083 & $0,10-0,125$ & 7,35 \\
\hline MAC & 0,1717 & 0,0356 & $0,13-0,25$ & 20,73 \\
\hline PC & 0,2783 & 0,0435 & $0,23-0,35$ & 15,63 \\
\hline LC & 0,2442 & 0,0229 & $0,21-0,28$ & 9,38 \\
\hline ARC & 76,5 & 3,3292 & $71-81$ & 4,35 \\
\hline
\end{tabular}

Tabla 1. Medidas de Cyclopelta winteri. La explicación de las abreviaturas se encuentra en el subapartado Caracteres Utilizados. Las medias y rangos están en $\mathrm{mm}$, excepto para el carácter $\mathrm{ARC}$, en grados sexagesimales. El coeficiente de varianza está expresado en $\%$.

inmediatamente distales mediante una expansión adelgazada del esqueleto laminar (Lám. I, fig. 3). Dos hileras de zoecios en cada rama, separados por una pared media en zigzag. Ramas y zoecios correspondientes ensanchados notablemente en los puntos de inserción de los disepimentos. Quilla en el anverso alta, con núcleo granular sinuoso en la base (en sección tangencial), volviéndose recto progresivamente hacia el extremo, que aparece bifurcado asimétricamente. Núcleo granular recubierto por esqueleto laminar, pudiendo éste espesarse sobre la bifurcación hasta producir un perfil convexo. Quillas unidas a la altura de la bifurcación por puentes de entre 0,10 y $0,25 \mathrm{~mm}$ de ancho (Lám. I, figs. 1, 2) que delimitan vanos similares a fenéstrulas, de 0,40 a 0,78 $\mathrm{mm}$ de longitud. Esta superestructura se observa a lo largo de todo el zoario, siendo el espesamiento del esqueleto laminar más acusado en la región proximal. Zoecios en dos hileras alternantes. Cámaras zoeciales pentagonales alargadas en sección tangencial profunda; pentagonales o hexagonales aproximadamente regulares o más anchas que largas en zonas de inserción de disepimentos. Paredes zoeciales transversales normalmente inclinadas en sentido distal, raramente verticales o inclinadas proximalmente. Cámaras entre disepimentos menos profundas y con base más plana que las situadas en zonas de inserción de disepimentos. Hacia el anverso, las cámaras se estrechan y tienen sección fabiforme, acabando en un tubo distal corto, inclinado levemente hacia las fenéstrulas. Aberturas zoeciales circulares, ligeramente elevadas sobre la superficie de la rama. Lámina basal delgada y cóncava; plana y continua cuando la sección transversal se realiza a lo largo de un disepimento (Lám. I, fig. 2). Los valores de los caracteres morfométricos de $C$. winteri se encuentran en la Tabla 1.

\section{Descripción}

C. winteri procede del Eifeliense de Gerolstein, Alemania.

\section{Discusión}

Cyclopelta winteri, al igual que otras especies de fenestrados, posee disepimentos oblicuos al plano del zoario, por lo que la anchura de disepimento (AD) debería ser medida en sección longitudinal y no en sección tangencial o en superficie, donde se obtienen valores inferiores a los reales. La asignación del material estudiado a Cyclopelta winteri se ha realizado basándose en la descripción y medidas publicadas por Kräusel (1953). La morfología zoarial, la asimetría de las quillas y la existencia de puentes conectando las mismas, así como la semejanza de valores de los caracteres morfométricos justifican esta determinación. La anchura de las ramas, distancia entre centros de ramas y número de fenéstrulas en $10 \mathrm{~mm}$ son ligeramente mayores en los especímenes asturianos. El número de ramas en $10 \mathrm{~mm}$ es similar si se traduce por $(10 \mathrm{~mm}-\mathrm{AR} / 2) / \mathrm{DCR}$ o por $(10$ $\mathrm{mm}$ - AR)/DCR. Los valores de LC y MAC son parecidos y el número de zoecios en $5 \mathrm{~mm}$ coincide si se traduce por $5 \mathrm{~mm} / \mathrm{DAH}$. McKinney y Kříž (1986) describen tres especies de Cyclopelta del Devónico Inferior de Bohemia; los especímenes asturianos se distinguen claramente de ellas por la morfología zoarial y la presencia de puentes uniendo las quillas.

\section{Lámina II}

1-3 Semicoscinium cf. discretum (Prantl, 1932). PIC-69-4; 1: sección tangencial profunda mostrando macroestiletes en el reverso de las ramas; x28. 2: sección transversal; se observa ensanchamiento apical de las quillas por esqueleto laminar, x28. 3 : sección longitudinal mostrando las indentaciones de la quilla, coincidentes con las aberturas zoeciales; $\mathrm{x} 28$.

4 Semicoscinium cf. discretum PIC-30-6; sección tangencial cortando la parte alta de las quillas; se aprecian pequeños estiletes a ambos lados de la pared granular; x26.

5-8 Hemitrypa sp. A; PIC-33-44, x26. 5: sección tangencial superficial, mostrando la forma de las celdillas en la superestructura. 6: sección tangencial profunda cortando ramas y los nodos que soportan la superestructura (parte inferior derecha). 7: sección transversal; la superestructura está casi totalmente erosionada; se observa la disposición de una abertura zoecial. 8: sección longitudinal; el reverso de la rama está silicificado y las cámaras se distinguen con dificultad. Hacia el centro de la fotografía se observan las paredes de la superestructura, coincidiendo las celdillas con aberturas zoeciales. 
Lámina II
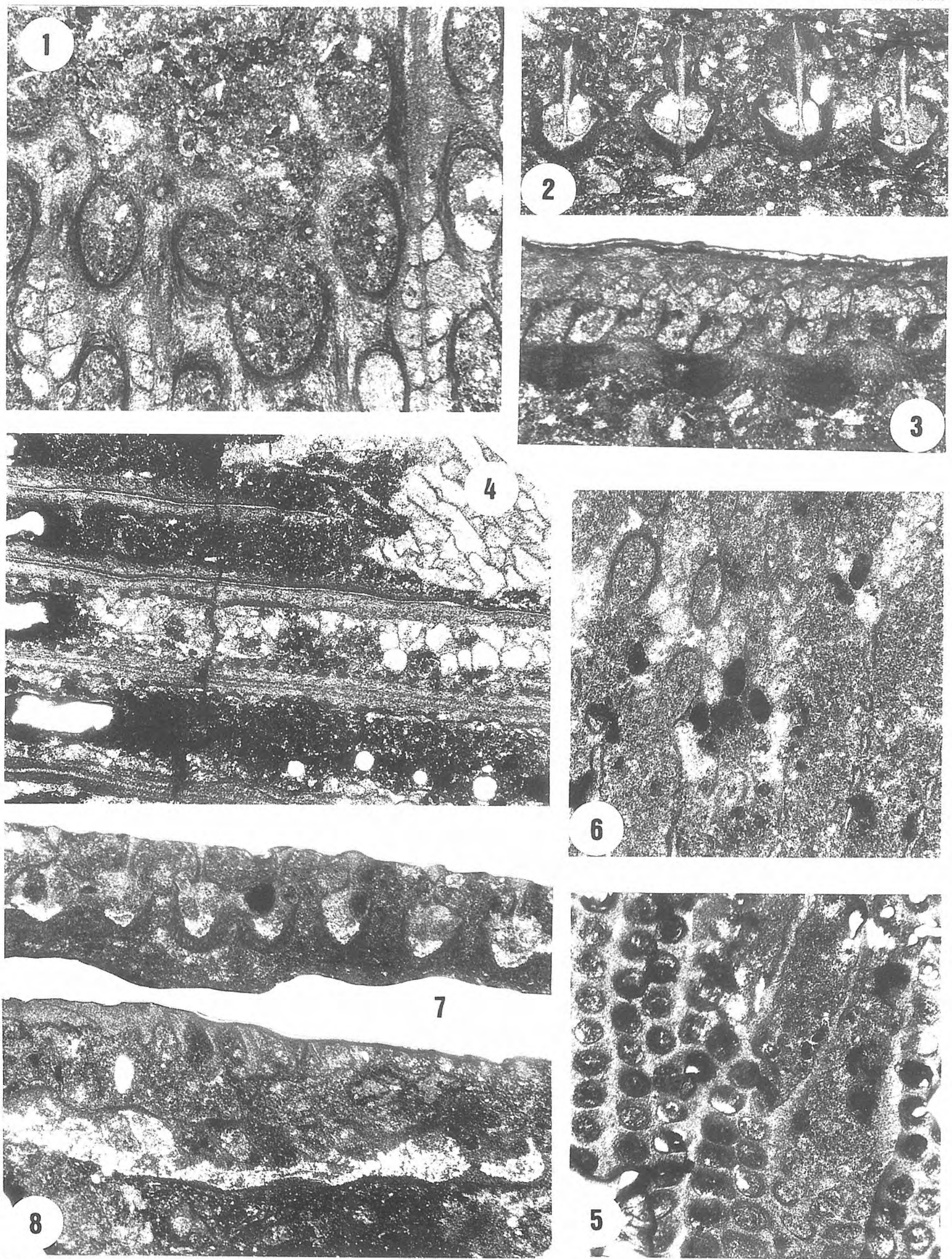

Revista Española de Paleontología, 13 (2), 1998.

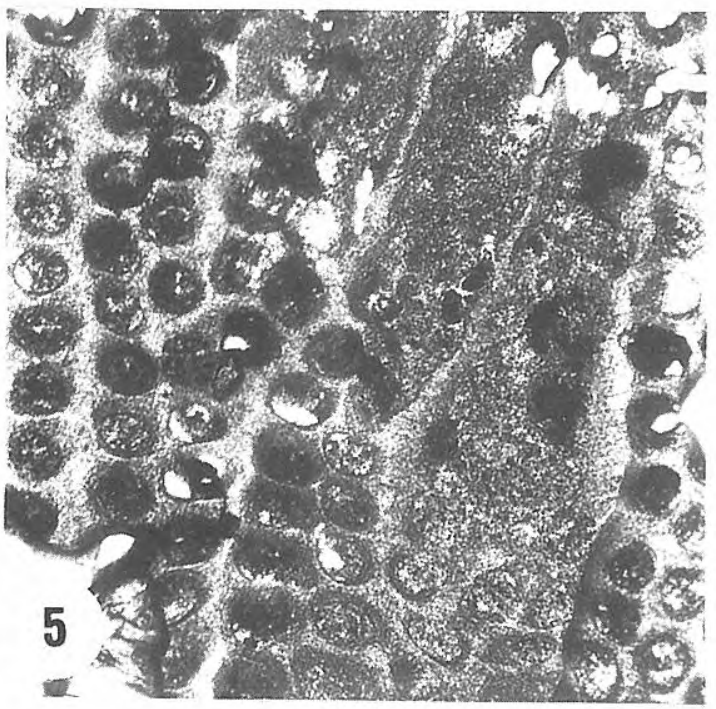




\section{Cyclopelta sp. A}

Lám. I, figs. 4-7

\section{Descripción}

Zoario en forma de embudo abierto con repliegues longitudinales más o menos abundantes. Ramas rectas a ligeramente sinuosas con dos hileras alternantes de autozoecios. Disepimentos cortos y anchos alineados transversalmente a las ramas, ligeramente deprimidos en el anverso y espesados hacia el reverso hasta unirse sobre las ramas formando anillos concéntricos. Sección transversal de los disepimentos elíptica o con forma de lágrima, inclinados hacia el reverso en sentido distal (Lám. I, fig. 7). Fenéstrulas ovales a elípticas, moderadamente elongadas, cuyas dimensiones se reducen hacia el reverso debido a la morfología de los disepimentos. Tejido vesicular presente en el anverso recubriendo disepimentos y obstruyendo parcialmente las fenéstrulas (Lám. I, figs. 4, 6). Quilla alta con núcleo granular en forma de "Y" simétrica y esqueleto laminar espesado, especialmente alrededor de la bifurcación. Ensanchamiento acusado de ramas y zoecios en las inserciones de disepimentos. Zoecios subpentagonales a subrectangulares entre disepimentos, pentagonales o hexagonales en las uniones con disepimentos (Lám. I, fig. 4). Pared axial en zigzag. Cámaras fabiformes en sección tangencial más superficial, acabando en tubos distales cortos con aberturas subcirculares. Lámina basal fina y cóncava entre disepimentos, plana a lo largo de los mismos. En este caso aparecen abundantes proyecciones que recuerdan microestiletes en el reverso de dichos disepimentos, manifestándose en superficie (Lám. I, fig. 5). Los valores de los caracteres morfométricos de Cyclopelta sp. A se encuentran en la Tabla 2.

\section{Discusión}

Aún no se han encontrado zoarios completos de Cyclopelta sp. A, aunque las dimensiones y curvatura de los fragmentos parecen indicar morfologías de tipo embudo o abanico de gran tamaño con repliegues. Cyclopelta sp. A presenta algunas diferencias con Cyclopelta winteri del mismo afloramiento: quillas simétricas y más estrechas, desprovistas de puentes, disepimentos ligeramente más anchos, ramas más separadas y dimensiones zoeciales mayores, si bien para los caracteres cuantitativos hay que tener en cuenta la provisionalidad de los resultados.

\section{Semicoscinium Prout, 1859}

Especie tipo: Semicoscinium rhomboideum Prout, 1859.

\section{Diagnosis}

Zoario en forma de embudo, aberturas zoeciales en la superficie externa. Ramas ligeramente sinuosas a anastomosadas, con dos hileras de zoecios. Quilla elevada con núcleo granular generalmente expandido hacia el extremo y esqueleto laminar comúnmente festoneado, coincidiendo las indentaciones con aberturas zoeciales. Cámaras rectangulares o cuadradas, con

\begin{tabular}{|l|l|l|l|l|}
\cline { 2 - 5 } \multicolumn{1}{c|}{} & media & desviación & rango & cf.varianza \\
\hline ER & 1,1225 & 0,0777 & $1,00-1,20$ & 6,92 \\
\hline AR & 0,4158 & 0,0835 & $0,35-0,65$ & 20,08 \\
\hline LF & 0,6300 & 0,0805 & $0,51-0,76$ & 12,77 \\
\hline AD & 0,8033 & 0,1181 & $0,63-1,07$ & 14,70 \\
\hline DCR & 0,6998 & 0,0819 & $0,55-0,83$ & 11,70 \\
\hline DAH & 0,2838 & 0,0167 & $0,25-0,32$ & 5,88 \\
\hline DAR & 0,3242 & 0,0314 & $0,28-0,38$ & 9,68 \\
\hline AA & 0,1446 & 0,0056 & $0,13-0,15$ & 3,87 \\
\hline MAC & 0,2142 & 0,0490 & $0,15-0,30$ & 22,88 \\
\hline PC & 0,3017 & 0,0279 & $0,25-0,34$ & 9,25 \\
\hline LC & 0,2500 & 0,0178 & $0,20-0,27$ & 7,12 \\
\hline ARC & 82,333 & 7,4870 & $70-90$ & 9,09 \\
\hline
\end{tabular}

Tabla 2. Medidas de Cyclopelta sp. A. La explicación de las abreviaturas se encuentra en el subapartado Caracteres Utilizados. Las medias y rangos están en $\mathrm{mm}$, excepto para el carácter ARC, en grados sexagesimales. El coeficiente de varianza está expresado en $\%$.

paredes laterales abombadas. Pared axial comúnmente recta, ocasionalmente sinuosa.

\section{Semicoscinium cf. discretum (Prantl, 1932)} Lám. I, fig. 8; Lám. II, figs. 1-4

\section{Descripción}

Zoario en forma de embudo ancho con pliegues longitudinales; aberturas zoeciales en la superficie externa. Ramas rectas o ligeramente sinuosas, incluso en un mismo zoario. Dos hileras de zoecios separadas por una pared axial recta (Lám. I, fig. 8), más raramente en leve zigzag, prolongada hacia el anverso en una quilla muy alta. Núcleo granular de la quilla en forma de lanza; envuelta laminar espesa, especialmente hacia el extremo, donde aparece festoneada (Lám. II, figs. 2, 3). En el techo de la quilla, dos hileras de macroestiletes alojados en el esqueleto laminar, separadas por el esqueleto granular (Lám. II, fig. 4). Reverso de las ramas con esqueleto laminar moderadamente espesado. Macroestiletes grandes, uniseriados, emplazados en el reverso aproximadamente en las zonas de inserción de los disepimentos, donde se produce un ensanchamiento moderado de las ramas y zoecios correspondientes (Lám. II, fig. 1). Disepimentos cortos y moderadamente anchos, subcirculares, redondeados hacia el anverso y más agudos hacia el reverso. Fenéstrulas elípticas anchas (hasta $0,4 \mathrm{~mm}$ ), excepto las distales a bifurcaciones, que son muy estrechas (0,20-0,25 mm). Cámaras zoeciales rectangulares o cuadradas en sección tangencial profunda (Lám. I, fig. 8), con paredes transversales formando un ángulo de $60^{\circ}$ con la 


\begin{tabular}{|l|l|l|l|l|}
\cline { 2 - 5 } \multicolumn{1}{c|}{} & media & desviación & rango & cf. varianza \\
\hline ER & 0,9158 & 0,0373 & $0,85-1,00$ & 4,07 \\
\hline AR & 0,3850 & 0,0653 & $0,30-0,50$ & 16,96 \\
\hline LF & 0,6425 & 0,0499 & $0,55-0,72$ & 7,77 \\
\hline AD & 0,4075 & 0,0685 & $0,32-0,52$ & 16,81 \\
\hline DCR & 0,6333 & 0,1434 & $0,50-0,92$ & 22,64 \\
\hline DAH & 0,2350 & 0,0194 & $0,20-0,27$ & 8,25 \\
\hline DAR & 0,3242 & 0,0390 & $0,25-0,40$ & 12,03 \\
\hline AA & 0,1083 & 0,0099 & $0,09-0,12$ & 9,14 \\
\hline MAC & 0,1600 & 0,0274 & $0,12-0,21$ & 17,12 \\
\hline PC & 0,2313 & 0,0153 & $0,21-0,26$ & 6,61 \\
\hline LC & 0,2383 & 0,0251 & $0,21-0,30$ & 10,53 \\
\hline ARC & 61,583 & 4,9575 & $53-70$ & 8,05 \\
\hline
\end{tabular}

Tabla 3. Medidas de Semicoscinium cf. discretum. La explicación de las abreviaturas se encuentra en el subapartado Caracteres Utilizados. Las medias y rangos están en mm, excepto para el carácter ARC, en grados sexagesimales. El coeficiente de varianza está expresado en \%.

axial en sentido distal. Cámaras profundas con tubo distal corto y ancho, terminado en abertura subcircular inclinada hacia la fenéstrula, con peristoma poco prominente. Tercer zoecio único en las bifurcaciones, romboide en sección tangencial. Lámina basal fuertemente cóncava de la que se proyectan hacia el reverso estrías longitudinales moderadamente gruesas (Lám. II, fig. 2). Los valores de los caracteres morfométricos de Semicoscinium cf. discretum se encuentran en la Tabla 3.

\section{Discusión}

El material estudiado se asemeja a Semicoscinium discretum (Prantl) McKinney y Kř́íz, 1986 en la morfología de la quilla, las ramas y las cámaras zoeciales, si bien el tubo distal parece estar más inclinado hacia la fenéstrula en los especímenes asturianos. Los valores provisionales obtenidos para los caracteres morfométricos son también comparables a los de Semicoscinium discretum, salvo para la distancia entre centros de aberturas en hilera, ligeramente mayor en aquella. Por otra parte, S. discretum (Prantl) aparece en el Zlichoviense (Emsiense), mientras que S. cf. discretum alcanza el Eifeliense en la sección muestreada.

\section{Hemitrypa Phillips, 1841}

Especie tipo: Hemitrypa oculata Phillips, 1841.

\section{Diagnosis}

Zoarios cónicos o en forma de abanico, constituidos por ramas con dos hileras alternantes de zoecios.
Disepimentos regularmente espaciados. Quilla en el anverso con espinas sobre las que se asienta una superestructura en forma de panal, en la que cada hueco corresponde a una abertura zoecial. Espinas con núcleo granular continuo con la pared media, y envuelta laminar. Cámaras zoeciales cuadrangulares o pentagonales irregulares en sección tangencial profunda, más o menos infladas lateralmente, con tubo distal corto.

\section{Discusión}

La presencia o ausencia de hemisepto es contradictoria en los trabajos de McKinney y Krríž (1986) y Snyder (1991a).

\section{Hemitrypa sp. A \\ Lám. II, figs. 5-8}

\section{Descripción}

Zoario en forma de embudo abierto con las aberturas zoeciales en el interior. Ramas por lo general rectas, pudiendo ser ligeramente sinuosas en algunos puntos del zoario. Dos hileras de zoecios por rama. Quilla recta con nodos uniseriados soportando la superestructura. Nodos en sección tangencial elípticos, moderadamente anchos, con núcleo granular y envuelta laminar atravesada por estiletes (Lám. II, fig. 6). Superestructura con quilla ancha y alta. Celdillas subpoligonales transversas, más o menos redondeadas por acumulación de esqueleto laminar; paredes inclinadas en sentido distal coherentemente con los tubos distales de los zoecios (Lám. II, figs. 5, 8). Esqueleto laminar espeso en el reverso de las ramas, con espesor máximo a lo largo de la línea media de la rama, produciendo una forma más o menos ojival en sección transversal. Microestiletes abundantes en el reverso. Ensanchamiento proximal y estrechamiento distal a bifurcaciones de ramas moderados a acusados. Disepimentos ovales angulosos o irregulares en sección longitudinal, inclinados de manera que apuntan distalmente hacia el reverso. Disepimentos alineados transversalmente a las ramas en áreas más o

\begin{tabular}{|l|l|l|l|l|}
\cline { 2 - 5 } \multicolumn{1}{c|}{} & media & desviación & rango & cf. varianza \\
\hline ER & 1,4370 & 0,0980 & $1,30-1,65$ & 6,80 \\
\hline AR & 0,3060 & 0,0490 & $0,24-0,38$ & 16,06 \\
\hline LF & 0,4330 & 0,0349 & $0,38-0,50$ & 8,07 \\
\hline AD & 0,2569 & 0,0300 & $0,20-0,31$ & 11,68 \\
\hline DCR & 0,5770 & 0,0657 & $0,45-0,70$ & 11,40 \\
\hline LRS & 0,1847 & 0,0173 & $0,15-0,21$ & 9,39 \\
\hline ARS & 0,2300 & 0,0247 & $0,19-0,27$ & 10,76 \\
\hline
\end{tabular}

Tabla 4. Medidas de Hemitrypa sp. A. La explicación de las abreviaturas se encuentra en el subapartado Caracteres Utilizados. Las medias y rangos están en $\mathrm{mm}$. El coeficiente de varianza está expresado en $\%$. 
menos extensas del zoario, del mismo modo que en Cyclopelta. En estas áreas, ramas y cámaras zoeciales pueden estar ensanchadas en los puntos de inserción de los disepimentos. Fenéstrulas elípticas moderadamente anchas, raramente subcirculares. Hileras de zoecios alternas, separadas por una pared sinuosa. Cámaras zoeciales pentagonales elongadas, aproximadamente equidimensionales en partes engrosadas de las ramas coincidentes con disepimentos alineados (Lám. II, fig. 6). En este caso el mayor tamaño de algunas cámaras posiblemente se deba a que funcionaran como ovicelos. Tubos distales inclinados distolateralmente, abriéndose hacia las fenéstrulas mediante aberturas con peristomas prominentes (Lám. II, fig. 7). Paredes zoeciales transversales inclinadas, tal que el tubo distal de un zoecio se apoya en la parte proximal del siguiente. Lámina basal cóncava desprovista de crestas longitudinales. Los valores de los caracteres morfométricos de Hemitrypa sp. A se encuentran en la Tabla 4.

\section{AGRADECIMIENTOS}

Al Dr. Soto, que ha dirigido la tesis de licenciatura de la que este trabajo representa una parte, y a los Dres. Bigey, Blake, Cuffey, Gilmour, Hageman, McKinney, Reguant, Snyder, Taylor y Tourneur por los consejos y la bibliografía que me proporcionaron, sin los cuales este estudio no hubiera sido posible. A las Doctoras Bigey y Méndez-Bedia, por sus comentarios, que han mejorado notablemente el manuscrito. Espero que este trabajo sea una muestra de agradecimiento hacia todos ellos.

\section{BIBLIOGRAFÍA}

Álvarez-Nava, H. y Arbizu, M. 1986. Composición y desarrollo de un arrecife emsiense en la Plataforma de Arnao (Asturias, NO. de España). Memorias I Jornadas de Paleontología, Zaragoza, 33-51.

Arbizu, M., García Alcalde, J. L., García Lopez, S., Méndez-Bedia, I., Sánchez de Posada, L. C., Soto, F. M., Truyols, M., Truyols, J., Álvarez, F., Méndez, C. \& Menéndez, J. R. 1979. Biostratigraphical Study of the Moniello Formation (Cantabrian Mountains, Asturias, NW Spain). A Contribution to the Lower/Middle Devonian Boundary Problem. Geologica et Palaeontologica, 13, 103-124.

Barrois, Ch. 1882. Recherches sur les terrains anciens des Asturies et de la Galice. Mémoires Societé Géologique du Nord, 2 (1), 1-630.

Boardman, R. S. \& Utgaard, J. 1964. Modifications of study methods for Paleozoic Bryozoa. Journal of Paleontology, 38, 768-770.

Bornemann, J. G. 1884. Cyclopelta Winteri, eine Bryozoe aus dem Eifeler Mitteldevon. Zeitschrift der deutchen geologischen gesellschaft, 36, 864-865.
Comte, P. 1959. Recherches sur les terrains anciens de la Cordillere Cantabrique. Memorias del Instituto Geológico y Minero de España, 60, 1-440.

García Alcalde, J. L. 1992. El Devónico de Santa María del Mar (Castrillón, Asturias, España). Revista Española de Paleontología, 7, 53-79.

García Alcalde, J. L. 1995. L’évolution paléogéographique pré-varisque de la Zone Cantabrique septentrionale (Espagne). Revista Española de Paleontología, 10, 9-29.

Hageman, S. J. 1991. Approaches to systematic and evolutionary studies of perplexing groups: an example using fenestrate Bryozoa. Journal of Paleontology, 65 , 630-647.

Julivert, M. 1967. La ventana del Río Monasterio y la terminación meridional del Manto del Ponga. Trabajos de Geología, 1, 59-76.

Kräusel, W. 1953. Cyclopelta winteri (Bryozoa) aus dem Mittel-Devon der Eifel. Senckenbergiana, 34, 43-52.

Kräusel, W. 1954. Cyclopelta aus dem corbis-Bank des Oberharzer Mittel-Devons. (Bryoz.). Senckenbergiana, 34, 246.

McKinney, F. K. \& Kříż, J. 1986. Lower Devonian Fenestrata (Bryozoa) of the Prague Basin, Barrandian Area, Bohemia, Czechoslovakia. Fieldiana Geology, 15, 1-90.

Méndez-Bedia, I. 1976. Biofacies y litofacies de la Formación Moniello-Santa Lucía (Devónico de la Cordillera Cantábrica, NO de España). Trabajos de Geología, 9, 1-93.

Phillips, J. 1841. Figures and Descriptions of the Palaeozoic Fossils of Cornwall, Devon and West Sommerset. Longmans, Brown, Green \& Longmans, London, 1-231.

Prantl, F. 1932. Revise Ceskych devonskych fenestellid. Palaeontographica Bohemiae, 15, 1-70.

Prout, H. A. 1859. Third series of descriptions of Bryozoa from the Paleozoic rocks of the western states and territories. Transactions of the Academy of Science of St. Louis, 1, 443-452.

Snyder, E. M. 1991a. Revised taxonomic procedures and paleoecologic applications for some North American Mississipian Fenestellidae and Polyporidae (Bryozoa). Palaeontographica Americana, 57, 1-275.

Snyder, E. M. 1991b. Revised taxonomic approach to acanthocladiid Bryozoa. In: Bryozoaires actuels et fossiles: Bryozoa living and fossil (Ed. F. P. Bigey). Bulletin de la Société des Sciences Naturelles de l'Ouest de la France, Mém. HS1, Nantes, 431-445.

Soto, F., Méndez-Bedia, I. y Fernández-Martínez, E. 1994. Construcciones arrecifales del Devónico de la Cordillera Cantábrica (NO de España). Revista Española de Paleontología, 9, 29-36.

Truyols, J., Arbizu, M., García Alcalde, J. L., García Lopez, S., Méndez-Bedia, I., Soto, F. \& Truyols-Massoni, M. 1990. Stratigraphy of the Asturian-Leonese Domain (Cantabrian Zone), In: Pre-Mesozoic Geology of Iberia (Eds. R. D. Dallmeyer \& E. Martínez García). Springer Verlag, Berlín, 10-19. 